Article

\title{
Vibrational Coupling of Nearest Neighbors in 1-D Spin Crossover Polymers of Rigid Bridging Ligands. A Nuclear Inelastic Scattering and DFT Study
}

\author{
Juliusz A. Wolny ${ }^{1, *}$, Isabelle Faus ${ }^{1}$, Jennifer Marx ${ }^{1}$, Rudolf Rüffer ${ }^{2}$, Aleksandr I. Chumakov ${ }^{2}$, \\ Kai Schlage ${ }^{3}$, Hans-Christian Wille ${ }^{3}$ and Volker Schünemann ${ }^{1}$ \\ 1 Department of Physics, University of Kaiserslautern, Erwin-Schrödinger-Str. 46, 67663 Kaiserslautern, \\ Germany; faus@rhrk.uni-kl.de (I.F.); marx@rhrk.uni-kl.de (J.M.); schuene@rhrk.uni-kl.de (V.S.) \\ 2 European Synchrotron Radiation Facility, CS 40220, Grenoble Cedex 9, 38043, France; ruffer@esrf.fr (R.R.); \\ chumakov@esrf.fr (A.I.C.) \\ 3 Deutsches Elektronen-Synchrotron DESY, Ein Forschungszentrum der Helmholtz-Gemeinschaft \\ Notkestraße 85, 22607 Hamburg, Germany; kai.schlage@desy.de (K.S.); \\ hans.christian.wille@desy.de (H.-C.W.) \\ * Correspondence: wolny@rhrk.uni-kl.de; Tel.: +49-631-205-4050
}

Academic Editors: Guillem Aromí and José Antonio Real

Received: 15 January 2016; Accepted: 25 February 2016; Published: 25 March 2016

\begin{abstract}
The nuclear inelastic scattering signatures of the low-spin centers of the methanosulphonate, tosylate, and perchlorate salts of the spin crossover polymer ([Fe(II)(4-amino-1,2,4-triazole $\left.\left.)_{3}\right]^{2+}\right)_{n}$ have been compared for the low-spin phase, for the mixed high-spin and low-spin phases, as well as for $\mathrm{Zn}$ (II) diluted samples. Within this series a change in the vibrational pattern in the 320-500 $\mathrm{cm}^{-1}$ region is observed. Significant shifts and decreasing intensity of bands at $\sim 320 \mathrm{~cm}^{-1}$ and bands over $400 \mathrm{~cm}^{-1}$ are observed as the molar fraction of the low-spin (LS) centers decrease. Density functional theory calculations using Gaussian09 (B3LYP/CEP-31G) for pentameric, heptameric, and nonameric model molecules yielded the normal modes of several spin isomers: these include the all high-spin (HS) and the all low-spin (LS) configuration but also mixtures of LS and HS centers, with a special focus on those with LS centers in a HS matrix and vice versa. The calculations reproduce the observed spectral changes and show that they are caused by strain extorted on a LS Fe(II) center by its HS neighbors due to the rigid character of the bridging aminotriazole ligand. Additionally, the normal mode analysis of several spin isomers points towards a coupling of the vibrations of the iron centers of the same spin: the metal-ligand stretching modes of the all LS and the all HS spin isomers reveal a collective character: all centers of the same spin are involved in characteristic normal modes. For the isomers containing both LS and HS centers, the vibrational behavior corresponds to two different subsets (sublattices) the vibrational modes of which are not coupled. Finally, the calculation of nuclear inelastic scattering data of spin isomers containing a ca. 1:1 mixture of HS and LS Fe(II) points towards the formation of blocks of the same spin during the spin transition, rather than to alternate structures with a HS-LS-HS-LS-HS motif.
\end{abstract}

Keywords: spin crossover; nuclear inelastic scattering; density functional theory

\section{Introduction}

The azole bridged Fe(II) 1D polymer complexes, such as the 1,2,4-triazoles [1,2] or alkylene-linked bitetrazoles, are of special importance for the investigation of the spin crossover (SCO) [3-6] effect. Designed over twenty years ago [7-9] with the idea of enhancing the cooperative character of the spin transition by linking the iron centers with a system of covalent bonds, these systems have revealed fascinating chemistry. They exhibit a very strong dependence of the spin transition temperature 
and the character of the transition on the type of the counter anions [2,10], the presence of hydrated water [11], and on the ligand substituent $[12,13]$. It has been shown that these SCO materials retain the cooperative character of their spin transition also as nanostructures [14-21]. This is important for the application of azole-bridged Fe(II)1D polymer complexes as materials for future molecular electronics and also provides an interesting challenge for theoretical modelling investigations. In spite of the new approaches based on different theoretical approaches involving molecular dynamics [22,23] the commonly-used measure of the cooperativity of a spin transition is the interaction parameter $\Gamma$, which on the theoretical level is derived on the basis of a phenomenological approach based on the mean-field approximation [24]. For the 1-D polymeric triazole ligand $\left[\mathrm{Fe}(\mathrm{Htrz})(\operatorname{trz})\left(\mathrm{BF}_{4}\right)\right]$ complex $[8,25]$ an interaction parameter $\Gamma$ of more than $10^{3} \mathrm{~cm}^{-1}$ has been reported [26,27], which is one order of magnitude larger than $\Gamma$ reported for molecular crystals of mononuclear Fe(II) SCO systems [24]. This indicates that, in 1-D polymers in which neighboring Fe(II) centers are linked by three rigid bridging ligands, the influence of the spin state of the neighbors on the spin transition energy of a given center is much higher than in molecular crystals of SCO complexes.

In order to get more insight into the microscopic nature of the cooperativity between the spin centers we started to perform density functional theory (DFT) calculations for a variety of spin isomers of increasing complexity. On the basis of previous work using trimeric and pentameric model molecules of the 4-amino-1,2,4-triazole (atrz) complex of Fe(II) [28] we are now extending our discussion to the properties of heptameric and nonameric model molecules. As an overview, the corresponding models used in the present work are shown in Scheme 1.

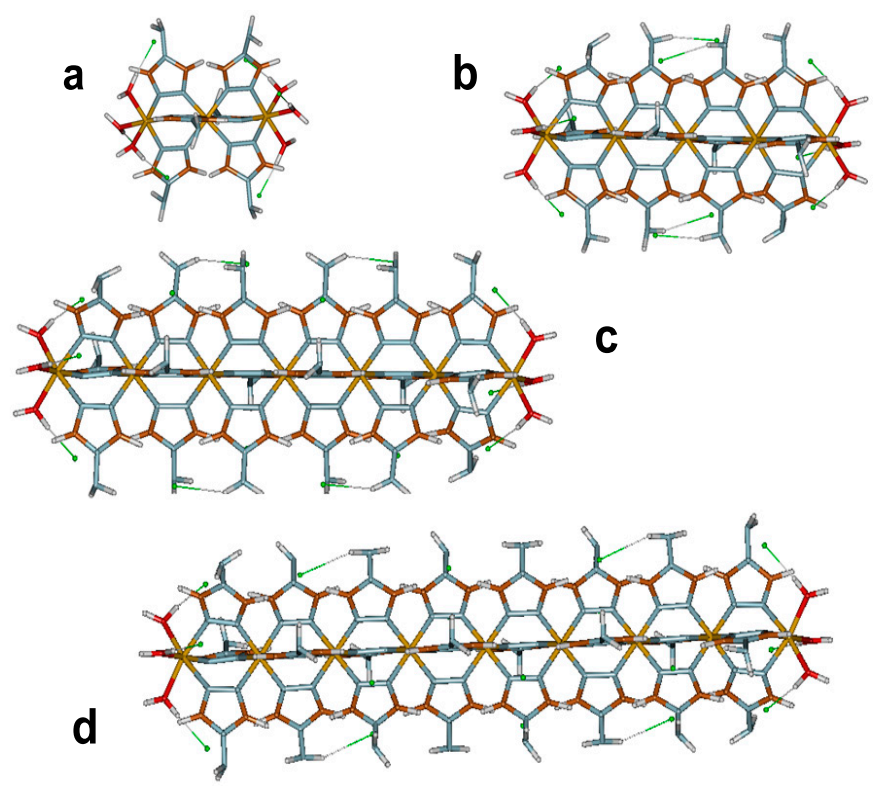

Scheme 1. Trimeric $\mathrm{Fe}_{3}(\operatorname{atrz})_{6}\left(\mathrm{H}_{2} \mathrm{O}\right)_{6} \mathrm{Cl}_{6}$ (a); pentameric $\mathrm{Fe}_{5}(\text { atrz })_{12}\left(\mathrm{H}_{2} \mathrm{O}\right)_{6} \mathrm{Cl}_{6}$ (b); heptameric $\mathrm{Fe}_{7}(\text { atrz })_{18}\left(\mathrm{H}_{2} \mathrm{O}\right)_{6} \mathrm{Cl}_{6}$ (c); and nonameric $\mathrm{Fe}_{9}(\text { atrz })_{24}\left(\mathrm{H}_{2} \mathrm{O}\right)_{6} \mathrm{Cl}_{6}(\mathbf{d})$ model molecules used in calculations in [28] (trimeric and pentameric) and in this study (heptameric and nonameric). In each case, the two terminal iron centers, with three coordinated waters are assumed to be in the high-spin state. All other centers may be either low-spin (denoted as L) or high-spin (denoted as H). Thus, for example, for the pentameric model five spin isomers are possible, denoted as HHHHH, HLLLH, HHLHH, HLHLH and HHLLH (identical with HLLHH). The iron in the inversion center is denoted as $\mathrm{Fe}(1)$, the next centrosymmetrically related ones are denoted as $\mathrm{Fe}(2) / \mathrm{Fe}\left(2^{\prime}\right), \mathrm{Fe}(3) / \mathrm{Fe}\left(3^{\prime}\right)$, etc.

Within all our models the terminal iron centers have a $\mathrm{N}_{3} \mathrm{O}_{3}$ coordination. Therefore, the spin state of the terminal iron is always HS (cf. [1]). In order to simplify the DFT calculations all the models discussed here are centrosymmetric and contain an odd number of iron centers unless stated otherwise. 
It is worth to note that the calculated NIS spectra and the principal geometrical parameters do not differ significantly if calculated for a pentameric, or an even numbered hexameric, model molecule [29].

We have shown before [28] that the rigid amino-triazole ligand is misdirected when it bridges two iron ions of different spin. The consequence is that the presence of the HS neighbors leads to an elongation of the $\mathrm{Fe}-\mathrm{N}$ distances for a given center, compared to the situation when the center has LS neighbors. Thus, a LS center which can be regarded as a "defect" in a HS matrix has longer $\mathrm{Fe}-\mathrm{N}$ distances than it has in a matrix which is in the all LS configuration. On the other hand the HS "defect" in a LS matrix will be compressed, compared to the all HS configuration. This effect leads to the destabilization of the LS state in a HS matrix and to a corresponding destabilization of the HS state in a LS matrix, compared to their "same spin" environments. The calculated differences of the LS $\rightarrow$ HS transition energies for the LS and HS nearest-neighbors was found to be always positive, varying from 15-35 kJ/mol depending on the applied model and exchange-correlation functional [28]. On the other hand, the calculated temperature dependencies of the vibrational contribution to the entropy for the LS $\rightarrow$ HS transition in the presence of neighbors with different spin imply that entropic effects act against the enthalpic stabilization of the LS state by the LS host. Furthermore, it was found that the difference of the vibrational entropies $\Delta S_{\text {vib }}$ for a transition e.g., in a pentameric chain from the HS-LS-LS-LS-HS to the HS-LS-HS-LS-HS state (furthermore denoted as: HLLLH $\rightarrow$ HLHLH) is about twice as large as for the HHLHH $\rightarrow \mathrm{HHHHH}$ transition where the switching center has HS neighbors. We have interpreted this effect as the result of a softening of LS vibrational modes in the HS matrix and the corresponding hardening of the HS modes in the LS matrix. This effect is a result of the elongation with respect to shortening of the Fe-N bonds, as mentioned above [28].

Here we present a detailed experimental and theoretical study of the iron ligand vibrations of the 4-amino-1,2,4-triazole bridged Fe(II) 1-D polymers for which the all LS phase, a mixture of the HS and the LS phase and specific LS centers in the matrix of HS centers could be observed. Nuclear inelastic scattering (NIS) of ${ }^{57} \mathrm{Fe}$ also called nuclear resonance vibrational spectroscopy (NRVS) is particularly well suited to study the vibrational properties of Fe(II) SCO systems [30,31]. This synchrotron-based technique uses the ${ }^{57} \mathrm{Fe}$ nucleus as a nuclear probe to detect vibrational modes, which include iron movement. NIS has no optical selection rules, the only requirement is that the vibrational mode involves the displacement of the ${ }^{57} \mathrm{Fe}$ site. The first results, including an initial modeling with a pentameric model, have been presented as a conference paper [32]. A previous NIS study of $\mathrm{Fe}(\mathrm{atrz})_{3}{ }^{2+}$ complexes revealed that the HS and LS marker bands are clearly distinct and rich in spectral features, but their calculated frequencies are quite independent on the type of anion used in the trimeric and pentameric models [29]. Within the present work we extend the DFT calculations to heptameric and nonameric models, that allow to investigate more spin isomers than the pentameric ones and correspond better to the 1-D polymers of Fe(II) complexes. On the basis of DFT calculations we present calculated vibrational partial density of states (pDOS), which are compared to the experimentally obtained pDOS of three samples: (i) The methanosulphonate salt of $\mathrm{Fe}(\mathrm{atrz})_{3}{ }^{2+}$ (atrz = 4-amino-1,2,4-triazole), denoted as (1), which is completely LS at $T=80 \mathrm{~K}$; (ii) the tosylate salt of Fe(atrz) ${ }_{3}{ }^{2+}(2)$ that reveals a hysteretic transition with $T_{\mathrm{c}}$ between 273 and $288 \mathrm{~K}$. For this sample we observe an approximate 1:1 mixture of LS and HS sites around $273 \mathrm{~K}$; (iii) the same salt diluted to $10 \%$ in the matrix of a zinc analogue (3). At $80 \mathrm{~K}$ this sample has LS centers which have predominantly $\mathrm{Zn}^{2+}$ centers as neighbors to the iron centers. We have chosen $\mathrm{Zn}^{2+}$, because it is considered to be a structural analogue of HS Fe(II) [3].

\section{Materials and Methods}

Samples (1) and (2) were prepared using standard methods as described in $[29,33]$, respectively. (3) was prepared analogously to (1), using a 1:9 mixture of ${ }^{57} \mathrm{Fe} / \mathrm{Zn}$ metanosulphonates. NIS was performed at the Nuclear Resonance Beamline ID 18 of the European Synchrotron Radiation Facility (ESRF) in Grenoble, France (see [32,33] for details) and at P01, PETRA III, DESY in Hamburg, Germany. The storage ring PETRA III was operated in 40 bunch mode with a bunch separation 
of 192 ns. DFT calculations were performed using the B3LYP* density functional $[34,35]$ and the CEP-31G basis set [36-38] as described in [28] using Gaussian09 Rev. D.01 [39] with full-charge compensation. For the penta- and heptameric models, the terminal iron centers were modeled as HS ones, with three coordinated water molecules. In this study we will use the previously [28] introduced notation of spin isomers. For example (see Scheme 1) HLLLH denotes the pentameric model molecule $\left(\mathrm{H}_{2} \mathrm{O}\right)_{3} \mathrm{Fe}(\mathrm{atrz})_{3}\left[\mathrm{Fe}_{3}(\mathrm{atrz})_{6}\right](\mathrm{atrz})_{3} \mathrm{Fe}\left(\mathrm{H}_{2} \mathrm{O}\right)_{3}$, with the three inner $\mathrm{Fe}(\mathrm{II})$ in the LS state and the two terminal Fe(II) centers in the HS state. In the case of nonameric models HHHHHHHHH, HHHHLHHHH and HLLLLHHHH the terminal HS centers were modeled with Zn(II) ions. For each model molecule frequency calculations were performed using the DFT-optimized structure. Unless indicated otherwise the calculated model molecules have $C_{i}$ symmetry. In each case the tight option of the integration grid was used. The simulated NIS spectra and the partial density of vibrational states of ${ }^{57} \mathrm{Fe}$ were obtained by using the program nisspec2 [40].

\section{Results and Discussion}

As described previously [32] the Mössbauer spectra of (1) revealed that all iron centers of this complex are in the LS state at $80 \mathrm{~K}$. On the contrary, the tosylate salt (2) showed $55 \%$ of LS and $45 \%$ of HS isomers at $273 \mathrm{~K}$, while the zinc-diluted sample (3) displays ca. $44 \%$ of LS and $56 \%$ of HS at $80 \mathrm{~K}$. The data for (1) and (2) are line with the magnetic susceptibility data reported previously for methanosulphonate of $\mathrm{Fe}(\mathrm{II})$ aminotriazole systems. The former reveals a hysteretic transition with $T_{\mathrm{c}} \downarrow$ of $\sim 260 \mathrm{~K}$ and $T_{\mathrm{C}} \uparrow$ of $\sim 295 \mathrm{~K}$ [41]. Other authors [2] quote $T_{\mathrm{c} \downarrow} \downarrow 273 \mathrm{~K}$ and $T_{\mathrm{c}} \uparrow \sim 288 \mathrm{~K}$. The tosylate salt reveals a hysteretic transition with $T_{\mathrm{c}} \downarrow \sim 279 \mathrm{~K}$ and $T_{\mathrm{c}} \uparrow \sim 296 \mathrm{~K}$ as detected by optical methods [42].

A comparison of the vibrational partial density of states (pDOS) of (1), (2) and (3) obtained from the NIS experiments in the frequency region where iron ligand vibrations occur are shown in Figure 1a-c. Although (2) and (3) reveal both a ratio of HS to LS centers of $c a$. 1:1 under the experimental conditions applied here, the LS iron centers in (3) are diluted to a molar ratio of 0.05 in a HS-like matrix of $\mathrm{Zn}^{2+}$ centers. Thus, (3) represents a sample which has LS iron centers as point "defects" in a HS matrix.

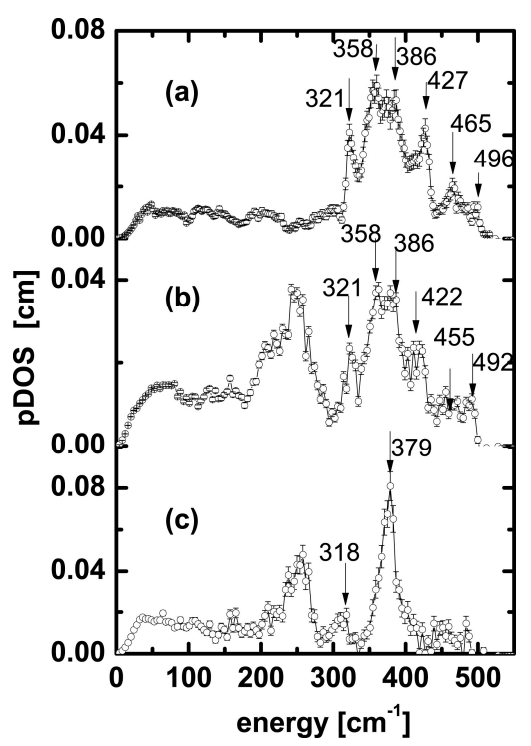

Figure 1. (a) Experimental pDOS of (1) obtained at $80 \mathrm{~K}$ (a); of (2) at $273 \mathrm{~K}$ (b); and of (3) at $80 \mathrm{~K}$ (c). Reprinted from [32].

Since the SCO transition from LS to HS for (1) and (2) occurs at about room temperature (with hysteresis), the pDOS shown in Figure 1a is characteristic for a $\left[\mathrm{Fe}(\operatorname{atrz})_{3}\right]^{2+}$ polymer with all iron 
sites being in the LS state and the pDOS shown in Figure $1 \mathrm{~b}$ reflects a $\left[\mathrm{Fe}(\operatorname{atrz})_{3}\right]^{2+}$ polymer with $\sim 50 \%$ HS iron sites and $\sim 50 \%$ LS iron sites. The pDOS of (3) shown in Figure 1c is also characteristic for a situation where $\sim 50 \%$ of the NIS-visible ${ }^{57} \mathrm{Fe}$ sites are in the LS state and $\sim 50 \%$ in the HS state, but these sites are separated from each other by the diamagnetic $\mathrm{Zn}$ (II) ions which act as NIS silent models of HS sites [3]. The inspection of Figure 1 shows that the band at $320 \mathrm{~cm}^{-1}$ is prominent in the pDOS of (1) (Figure 1a) but it has less intensity in the pDOS of (2) (Figure 1b) and in the Zn(II) diluted sample (3) (Figure 1c). The same trend is observed for bands in the region between 420 and $500 \mathrm{~cm}^{-1}$, for (3) these bands nearly vanish. These changes are concomitant with the decreasing probability that a LS center has LS neighbors within the 1-D polymer series (1), (2), and (3). There is also a concomitant change of the spectral pattern in the $350-390 \mathrm{~cm}^{-1}$ area-the relative broad band with at least two maxima at 358 and $386 \mathrm{~cm}^{-1}$ in (1) and (2) is replaced by a more narrow band with a maximum at $379 \mathrm{~cm}^{-1}$ in (3).

In order to account for these effects, the pDOS of selected molecular units has been calculated via DFT and subsequent normal mode analysis. For this purpose pentameric, heptameric, and nonameric model molecules of $C_{i}$ symmetry were used. Calculations investigating the effect of the number of LS sites on the overall pDOS are shown in Figure $2 b-d$ in comparison to the experimental pDOS (Figures 1a and 2a). Surprisingly, the reproducibility of the experimental pDOS by all three structural models is quite reasonable. However, the vibrational signature of the central $\mathrm{Fe}(1)$ shows a very significant effect on the number of neighboring LS sites (Figure 2e-g). In fact, the calculations performed with the nonameric model show a pDOS of the central Fe(1) (Figure 2g) which very much resembles the shape of the pDOS of the whole nonameric model displayed in Figure 1d.
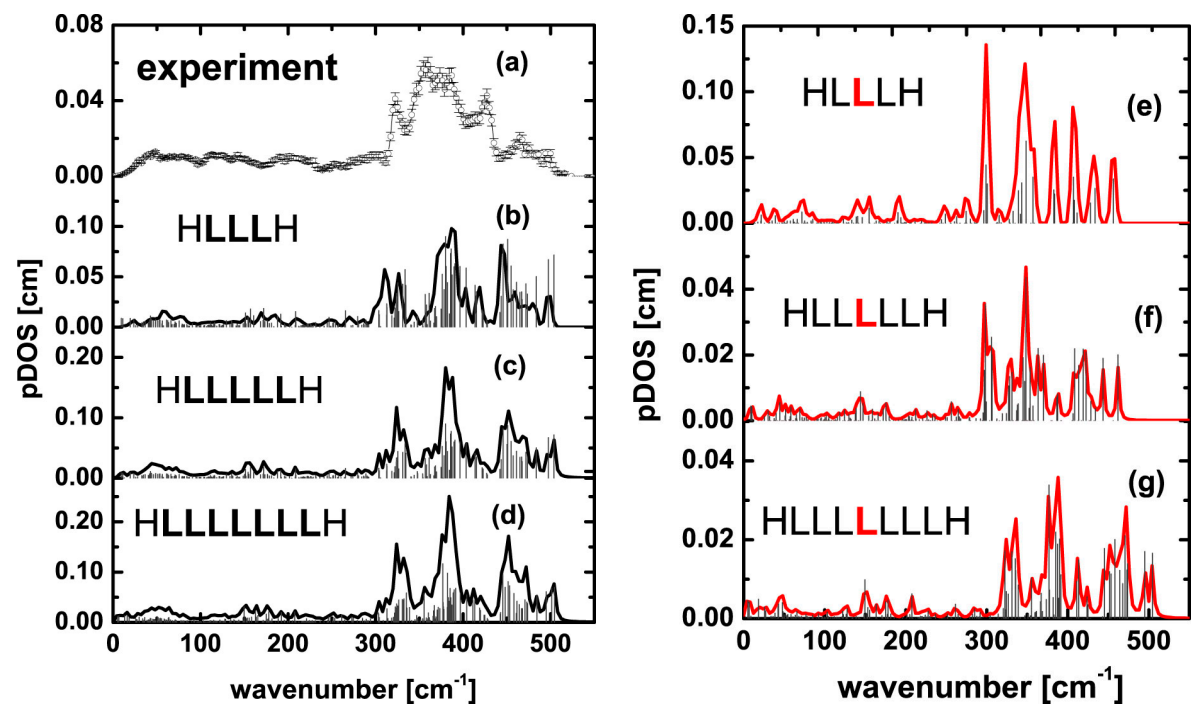

Figure 2. Left: Experimental pDOS of (1) (pure LS phase) (a) and calculated pDOS involving modes of all LS centers for the pentameric (b); heptameric (c); and nonameric (d) model molecules displayed in Scheme 1. Right: Simulated pDOS of only the central LS Fe(1) (red) calculated with the pentameric (e); heptameric (f) and nonameric (g) model molecules. The bars denote the calculated vibrational modes scaled to $1 / 5$ of their calculated intensity. The iron centers for which the pDOS has been calculated are marked in bold.

In order to get more insight into the observed shifts and intensity changes of the vibrational iron ligand modes we discuss in the following a few characteristic normal modes, calculated with pentameric, heptameric, and (in some cases) nonameric models. The modes are shown as movies in Supplementary Materials. The results for three bands observed in the NIS spectra of (1) are shown in Table 1. 
Table 1. Comparison of the energies $\left(\mathrm{cm}^{-1}\right)$ of three experimentally-observed vibrational bands in the pDOS of (1) and (3) and of the corresponding modes calculated with DFT for the different indicated models displayed in Scheme $1 \mathrm{~b}-\mathrm{d}$. The names of the corresponding movies showing a particular mode are given (cf. Supplementary Materials).

\begin{tabular}{|c|c|c|c|c|c|c|c|}
\hline \multicolumn{2}{|r|}{ Experiment } & \multicolumn{2}{|c|}{ Pentameric Model } & \multicolumn{2}{|c|}{ Heptameric Model } & \multicolumn{2}{|c|}{ Nonameric Model } \\
\hline \multicolumn{8}{|c|}{ Sample Spin of neighbours } \\
\hline LS (1) (1) & LS in Zn matrix (3) & LS & $\mathrm{HS}$ & LS & HS & LS & HS \\
\hline 321 & 318 & 311 (ls_pent_311) & $\begin{array}{c}302 \\
303(\mathrm{HHL}+\mathrm{HH} 303)\end{array}$ & $\begin{array}{c}324 \\
325(\text { s hent 325) }\end{array}$ & $\begin{array}{c}301 \\
305(\mathrm{HH} L \mathrm{HHH} 305)\end{array}$ & 327 (1s_non_327) & Not observed \\
\hline 465 & Weak peaks in $450-495 \mathrm{~cm}^{-1}$ region & $\begin{array}{l}326 \text { (Is_pent_326) } \\
467\end{array}$ & $\begin{array}{l}303 \text { (HHLH1_303) } \\
445\end{array}$ & $\begin{array}{l}325 \text { (IS_nept_325) } \\
461\end{array}$ & $\begin{array}{l}\text { 305 (HAHLFHH_3U5) } \\
448\end{array}$ & 471 (ls_non_471) & 435 (HHHHLHHHH_non_435) \\
\hline 496 & Weak peaks in $450-495 \mathrm{~cm}^{-1}$ region & 498 (ls_pent_498) & 461 & 504 & 460 (HHHLHHH_460) & 505 (ls_non_505) & 509 \\
\hline
\end{tabular}


We begin with the mode observed at $c a .320 \mathrm{~cm}^{-1}$ for LS (1) (see Figures 1a and 2a). For the pentameric model two NIS active vibrations are predicted in this area: Fe-N stretching of Fe(1) (the central one) at $326 \mathrm{~cm}^{-1}$ (movie ls_pent_326) and the corresponding vibration of centrosymmetrically located $\mathrm{Fe}(2)$ and $\mathrm{Fe}\left(2^{\prime}\right)$ at $311 \mathrm{~cm}^{-1}$ (movie ls_pent_311) (see Figure 2b). For the heptameric model the pDOS in this area is dominated by two vibrations occurring at 324 and $325 \mathrm{~cm}^{-1}$ which involve primarily Fe-N stretching of Fe(1) (movie ls_hept_325) (see Figure 2c). The nonameric model reveals the significant stretching of $\mathrm{Fe}(1), \mathrm{Fe}(2) /\left(2^{\prime}\right)$ and $\mathrm{Fe}(3) /\left(3^{\prime}\right) \mathrm{Fe}-\mathrm{N}$ at $327 \mathrm{~cm}^{-1}$ (movie ls_non_327) (see Figure 2a). What happens when all the neighbors of the central LS Fe(1) turn to the HS state? With the heptameric model, two LS Fe-N stretching vibrations are now predicted at 301 and $305 \mathrm{~cm}^{-1}$ (movie HHHLHHH_hept_305) (see Figure 3f), while the pentameric model yields two similar bands at 302 and $303 \mathrm{~cm}^{-1}$ (movie HHLHH_pent_303) (see Figure 3e). For a nonameric model no band in the pDOS can be found that corresponds to a vibration involving the LS Fe(1) in the region below $350 \mathrm{~cm}^{-1}$ (see Figure 3g). Accordingly, we explain the experimentally observed band shift of $c a .15 \mathrm{~cm}^{-1} \mathrm{by}$ the change of the bond lengths. Indeed, previously reported calculations reveal that the $\mathrm{Fe}(1)-\mathrm{N}$ bond lengths for a pentameric HLLLH isomer (B3LYP*/CEP-31G) are ca. $0.024 \AA$ shorter than for a HHLHH one, implying a possible shift of the stretching vibrations towards the lower frequencies for the latter.
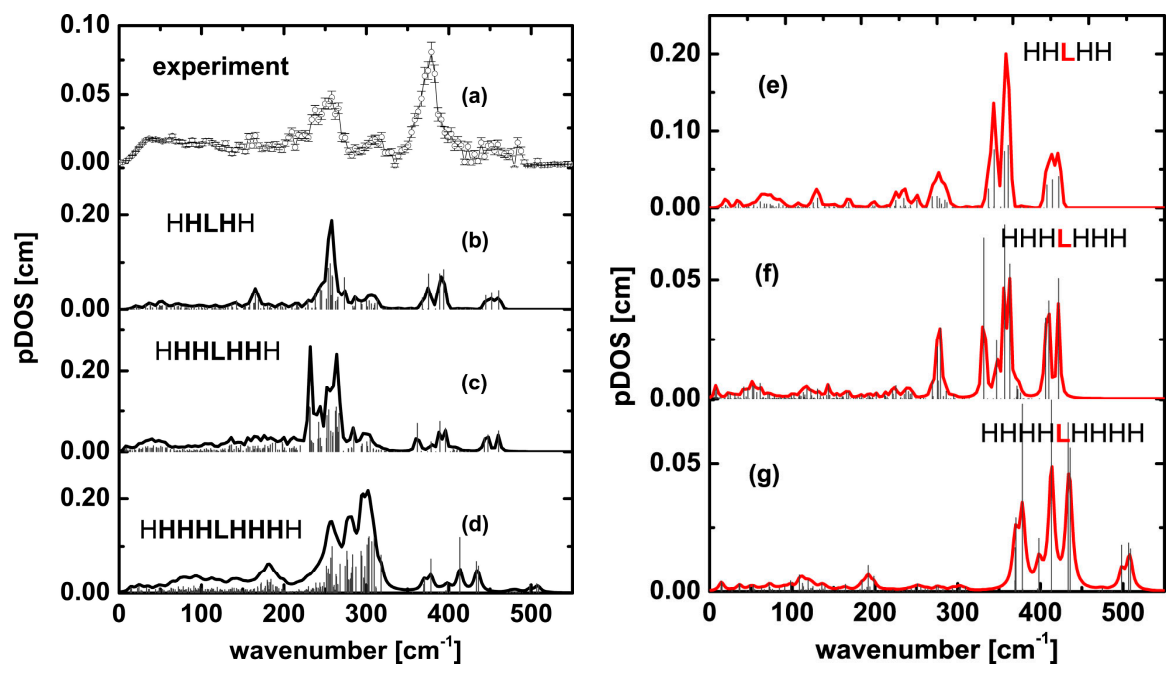

Figure 3. Left: Experimental pDOS of (3) (ca. 1:1 mixture of LS and HS Fe(II) centers, diluted in Zn(II) matrix) (a) and DFT simulations involving modes of all but the terminal centers for pentameric (b); heptameric (c); and nonameric (d) model molecules with LS Fe(II) in center and all HS neighbors. Right: Simulated pDOS of only the central LS Fe(1) (red) calculated with the pentameric (e); heptameric (f); and nonameric (g) model molecules. The centers taken for a given calculations of pDOS are marked in bold. The bars denote the calculated vibrational iron modes scaled to $1 / 5$ of their calculated intensity. The most intensive iron vibration of the heptameric and nonameric model are truncated for clarity reasons. The calculations shown left involved all HS Fe(II) neighbors, although the spectrum was taken for the $\mathrm{Zn}$ (II) diluted sample; therefore, the intensity of the bands at $200-300 \mathrm{~cm}^{-1}$ due to the HS vibrations is overestimated.

The next two bands that are influenced on changing from (1) to (3) are those at $496 \mathrm{~cm}^{-1}$ and $465 \mathrm{~cm}^{-1}$. The first one corresponds to the in-phase movement of all three LS Fe centers along the long axis of the molecule in the pentameric HLLLH model, predicted at $498 \mathrm{~cm}^{-1}$ (movie ls_pent_498) (see Figure 2b). The analogous mode, involving the movement of all five LS Fe center is predicted to occur at $504 \mathrm{~cm}^{-1}$ for the heptameric model, while the nonameric one predicts this mode at practically the same energy of $505 \mathrm{~cm}^{-1}$ (movie 1s_non_505) (see Figure 2c). Within the HS matrix, this LS vibration involves only the $\mathrm{Fe}(1)$ atom and does not involve the movement of the HS neighbors. It is predicted to occur at $461 \mathrm{~cm}^{-1}$ for the pentameric HHLHH model and at $460 \mathrm{~cm}^{-1}$ for the heptameric 
HHHLHHH (movie HHHLHHH_460) (see Figure 3e,f). In this case the observed shift of $\sim 40 \mathrm{~cm}^{-1}$ is, again, due to the differences in Fe-N bonds mentioned above. The pronounced change in the band intensity in the pDOS is related to a lower cumulative projection of the mean square displacement of the Fe atoms: the fewer iron atoms are involved in the stretching mode, the less displacement of the iron has been calculated.

The band observed at $463 \mathrm{~cm}^{-1}$ in (1) is assigned to an out-of-phase Fe-N stretching, perpendicular to the long axis, involving movement of $\mathrm{Fe}(1)$ and $\mathrm{Fe}(2) / \mathrm{Fe}\left(2^{\prime}\right)$ within the pentameric model. This mode is calculated to occur at $467 \mathrm{~cm}^{-1}$ for HLLLH (see Figure $2 \mathrm{~b}$ ). For the heptameric model the analogous vibration involves atoms $\mathrm{Fe}(3) / \mathrm{Fe}\left(3^{\prime}\right)$ and is predicted at $461 \mathrm{~cm}^{-1}$ (see Figure 2c), while the nonameric model has it at $471 \mathrm{~cm}^{-1}$ (see Figure 2d). Upon switching all Fe centers to the HS state except the Fe(1) centers, which stay LS, this vibration shifts to $445,448 \mathrm{~cm}^{-1}$ and $435 \mathrm{~cm}^{-1}$ in the pentameric, heptameric, and nonameric models, respectively (see Figure $3 \mathrm{e}-\mathrm{g}$ ).

Thus, the experimentally-observed changes of the spectral pattern of the LS marker bands going from the LS to HS/HS analogue matrix could be accounted for using the DFT modeling. This confirms the previously-predicted [28] strain extorted on a LS Fe(II) center by its HS neighbors due to the rigid character of the bridging aminotriazole ligand.

Another interesting observation is that for the above discussed modes a decoupling of Fe-N stretching of the LS centers and the HS matrix is observed: If surrounded by LS centers the modes involving the LS Fe(1) reveal a collective character, with all LS centers of the molecule moving. On the contrary, when the LS Fe(1) is surrounded by HS centers the corresponding mode does not involve any movement of the HS neighbors and only the LS Fe(1) is moving.

In the next step we will analyze the pDOS of (2). As shown previously, minor shifts (a few cm${ }^{-1}$ ) of bands of the LS isomer above $400 \mathrm{~cm}^{-1}$ are observed in comparison to the all LS (1). These could be interpreted by using the pentameric HLLHH model [32]. Here we present the results of DFT calculations based on the heptameric and the nonameric models. These allow the comparison of three distribution patterns of the LS and HS centers by keeping an effective LS:HS ratio of one. We discuss first the situation where both spin isomers are present in one chain, distributed according to two different patterns: (a) the one with all centers having neighbors of different spin-this pattern we denote as the "chessboard" (alternate) one, for example in a HLHLHLH isomer; and (b) the one with two blocks of the same spin-this pattern we denote as the "block" one, for example HLLLHHH or HHHHLLH isomers. However, there is also a possibility that a sample with a LS:HS ratio of one consists of a mixture of all LS and all HS chains. The pDOS of such a system would then correspond to the sum of the pDOS calculated for all LS and all HS chains. This situation can be modeled by taking the sum of the pDOS calculated i.e., for a HLLLLLH and a HHHHHHH heptameric models (or corresponding nonameric ones). In Figure 4 the comparison of the experimentally-determined pDOS of (2) with several models used for DFT calculations is shown.

The inspection of the results shown in Figure 4 reveals that the pDOS of the chessboard (see Figure $4 \mathrm{~b}, \mathrm{c}$ ) and block patterns (see Figure $4 \mathrm{~d}, \mathrm{f}$ ) are quite distinct. The comparison of them with the experimentally determined pDOS of (2) at $273 \mathrm{~K}$ suggests that the block model fits better to the experimental results. The HS marker bands occurring at $180-290 \mathrm{~cm}^{-1}$ are better reproduced by the block model, than by the chessboard one. For the block models they span the $210-290 \mathrm{~cm}^{-1}$ range, while for the checkerboard they are calculated to occur in the $230-320 \mathrm{~cm}^{-1}$ range. Additionally, the double peak character of this band cluster is better reproduced with the block model, particularly for the nonameric HLLLLHHHH model (see Figure 4f). On the other hand, the distribution of the bands of the LS center seems to be more evenly distributed for the block models compared to the checkerboard ones. Such an even distribution reflects better the character of the LS part of the experimentally determined pDOS of (2) at $273 \mathrm{~K}$ displayed in Figure 4a. 


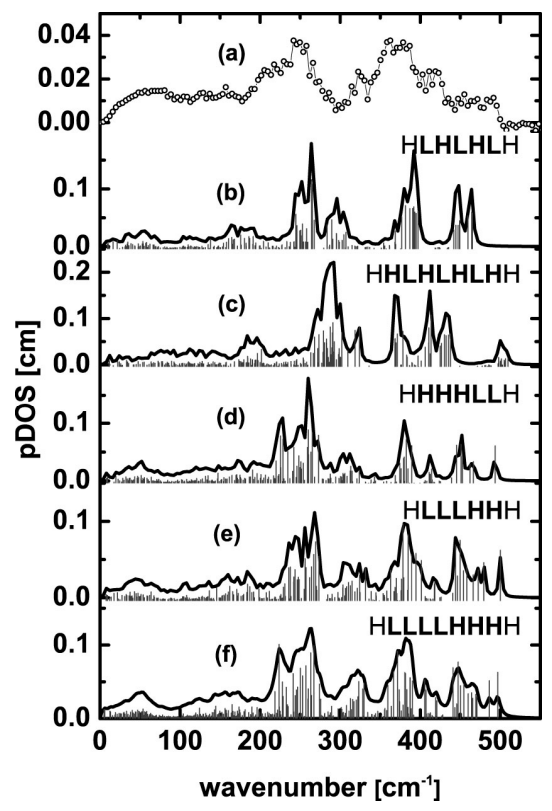

Figure 4. Comparison of the experimental pDOS of (2) at $273 \mathrm{~K}$ (a) with the calculated pDOS of the chessboard $(\mathbf{b}, \mathbf{c})$ and block models $(\mathbf{d}-\mathbf{f})$. The bars denote the calculated vibrational iron modes scaled to $1 / 5$ of their calculated intensity. Note that the applied models have a LS:HS ratio of 3:2, 2:3, and 4:3, rather than an exact $1: 1$ ratio.

The pDOS as obtained by taking the 1:1 sum of the all LS and the all HS pDOS of all three, pentameric, heptameric, and nonameric, models (see Supplementary Materials) are similar to those obtained for the block models as far as the pattern of the LS and HS marker bands are concerned. They seem to fit equally good the experimentally obtained pDOS. In summary, the pDOS of (2) obtained at a temperature close to $T_{\mathrm{C}}$ implies that the 1:1 mixture of LS and HS centers reveals a spectral pattern that indicates the clustering of the centers of the same spin. It is not characteristic for an alternate or a random distribution of HS and LS centers along the chain.

Furthermore, we have analyzed the influence of the spin of the neighbors on the vibrational properties of a single HS center. The experimental realization of a HS Fe(II) center in a matrix corresponding only to LS neighbors is fairly difficult; therefore, we present only the results of the DFT modeling. On the basis of previously obtained DFT results [28] one may expect that the predicted compression of the HS coordination sphere on going from a neighboring HS to a LS matrix results in a shift of the HS Fe(II)-N stretching modes to higher frequencies. Therefore, the calculations for the pentameric, heptameric, and nonameric pairs of pure HS isomers and isomers displaying a HS Fe(II) at the symmetry center in the environment of LS Fe(II) centers were performed. The calculated pDOS, which originates only from the central HS Fe(1) in its LS and its HS environments, are displayed in Figure 5.

The calculated pDOS displayed in Figure 5 clearly show changes in the vibrational modes when the spin of the neighbors change. The analysis of the modes exhibits that the relation between the frequency of a given mode of a HS Fe(1) and the spin of its neighbors is less clear than for the LS one, as discussed above. For example, the mode at $227 \mathrm{~cm}^{-1}$ for the HHHHH model shifts only to $235 \mathrm{~cm}^{-1}$ for HLHLH (see Figure 5, left) (compare the movies hs_pent_227 and HLHLH_pent_235). On the other hand the predicted mode at $160 \mathrm{~cm}^{-1}$ in HLHLH resembles that predicted at $128 \mathrm{~cm}^{-1}$ for HHHHH (cf. hs_pent_128 and HLHLH_pent_160). The mode predicted in the $181-187 \mathrm{~cm}^{-1}$ range for heptameric HLLHLLH corresponds to the band obtained for HHHHHHH (see Figure 5, middle) as low as at $120 \mathrm{~cm}^{-1}$ ( $c f$. movies hs_hept_120 and HLLHLLH_hept_184). Yet the mode at $273 \mathrm{~cm}^{-1}$ predicted for HHHHHHH that gives the most intensive NIS peak is comparable to both of those of HHHLHHH 
at $272 \mathrm{~cm}^{-1}$ and and $291 \mathrm{~cm}^{-1}$ ) (cf. movies hs_hept_273, HLLHLLH _hept_272, and HLLHLLH _hept_291). Additionally, all three models predict a disappearance of the peak at $310-320 \mathrm{~cm}^{-1}$, which is present in the all HS molecule, upon spin switching of the neighbors of $\mathrm{Fe}(1)$. This less clear picture may be due to a higher elasticity of the $\mathrm{HS} \mathrm{Fe}(\mathrm{II})-\mathrm{N}_{6}$ coordination core.
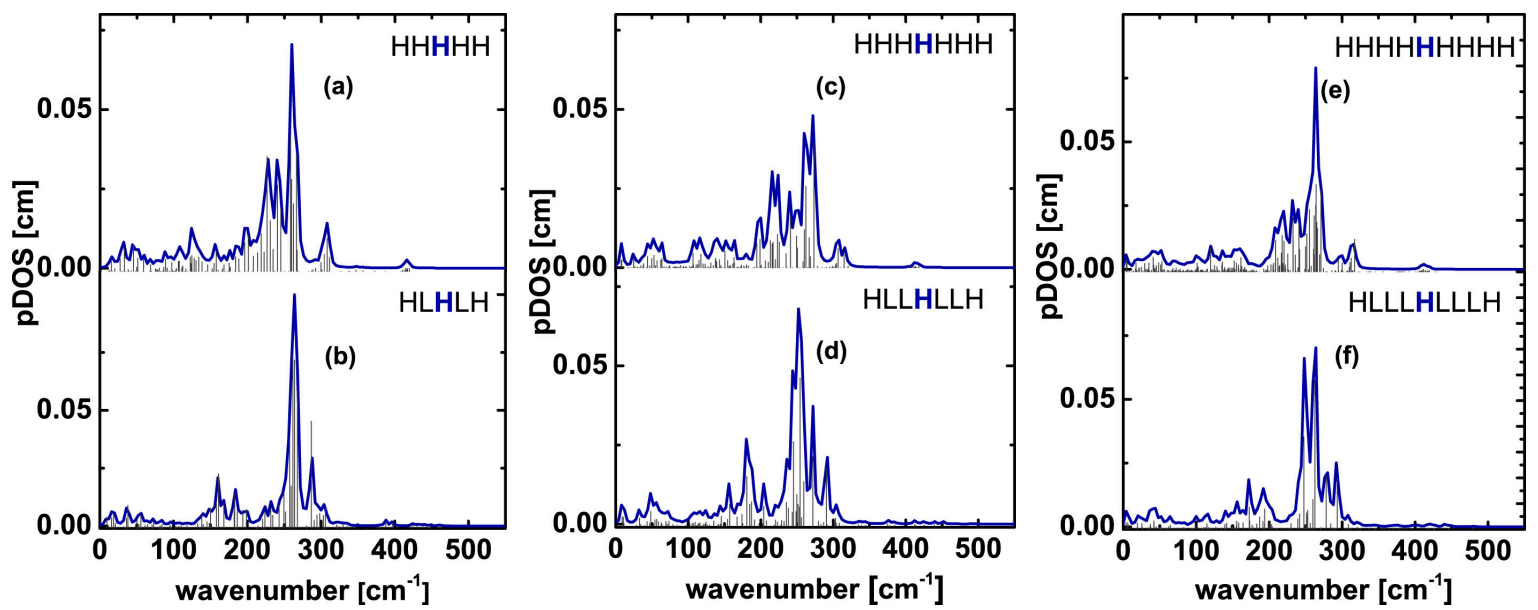

Figure 5. Calculated pDOS for HS Fe(1) (bold, highlighted blue) in HS (top) and LS (bottom) matrix for pentameric (left), heptameric (middle), and nonameric (right) models. The bars denote the calculated vibrational iron modes scaled to $1 / 5$ of their calculated intensity.

Finally, we discuss two examples of how the presence of centers of different spin in the 1-D chain leads to decoupling of the analogous modes. The Fe-N stretching along the long axis of the molecule in nonameric HHLHLHLHH is present in two different modes: (i) the one at $411 \mathrm{~cm}^{-1}$ (cf. movie non_chessb_411) involving only the LS centers and (ii) that at $318 \mathrm{~cm}^{-1}$ (cf. movie non_chessb_318) involving only HS centers. A similar effect is observed for the block structure isomer HLLLHHL, for which the correlated stretching of the above type is predicted at $389 \mathrm{~cm}^{-1}$, involving the LS ensemble while the most similar modes for the HS one could be found at 256 and $267 \mathrm{~cm}^{-1}$ (compare the movies hept_block_LLLHH_389/256/267). This effect suggests that in both alternate and in domain-like structures the two spin isomers form two independent subunits which can be regarded as "sublattices". Taking into account that according to theoretical models the spin crossover in solids takes place via interactions communicated through low energy lattice vibrations the similar decoupling for the acoustic phonons may be of importance for the character of the transition [43,44].

It is important to note at that the related effects have also been observed for 3-D polymeric $\mathrm{Fe}$ (pyrazine) $\left[\mathrm{Pt}(\mathrm{CN})_{4}\right]$ system diluted in $\mathrm{Ni}\left(\right.$ pyrazine) $\left[\mathrm{Pt}(\mathrm{CN})_{4}\right]$ and $\mathrm{Co}($ pyrazine $)\left[\mathrm{Pt}(\mathrm{CN})_{4}\right]$ matrices [45]. In this study it has been observed that low-frequency HS and LS Raman marker bands shift on increasing dilution of the spin crossover centers. The LS marker band at $120 \mathrm{~cm}^{-1}$ shifted to lower frequencies with decreasing molar fraction of iron, while an upshift of the HS band at $c a .50 \mathrm{~cm}^{-1}$ was observed.

The effects presented in this paper have consequences for the spin crossover entropy and its contribution to the cooperativity of the spin transition. The importance of the molecular vibrations for the spin crossover phenomenon is generally recognized [46]. The vibrational contribution to the entropy, which can be derived from vibrational spectra and DFT calculations $[30,46-48]$ is not only the additional driving force of the spin transition. Additionally, low energy phonons contribute to the cooperativity as suggested by theoretical considerations based on the Ising model [49] and on elasticity theory [50]. As already stated, we have, by use of the DFT procedure described in [47], previously calculated the vibrational contribution to the entropy for the HLLLH to HLHLH and the HHLHH to HHHHH transitions of the systems described here [28]. The results show that the vibrational entropy contribution, $\mathrm{S}_{\mathrm{vib}}$, is significantly higher for the first one. The entropy difference that we called $S_{\text {coop }}$ 
varies from $c a .20 \mathrm{~J} / \mathrm{K} \cdot \mathrm{mol}$ at $50 \mathrm{~K}$ to $c a .35 \mathrm{~J} / \mathrm{K} \cdot \mathrm{mol}$ at $400 \mathrm{~K}$. Thus, the LS neighbors destabilize the LS center entropically, while the HS ones stabilize it. It is interesting to compare this outcome and the here presented results with the predictions of an Ising-like model by Bousseksou et al. [51]. These authors define the vibrational coupling parameter $\eta$ as:

$$
\eta=\sum_{i=1}^{15} \ln \left(\frac{\left(\frac{\omega_{\mathrm{LS}}^{(i)}}{\omega_{\mathrm{HS}}^{(i)}}\right)_{\mathrm{HS}}}{\left(\frac{\omega_{\mathrm{LS}}^{(i)}}{\omega_{\mathrm{HS}}^{(i)}}\right)_{\mathrm{LS}}}\right)
$$

where the outer indices denote the spin state of the lattice and $\omega$ the frequency of the vibrational mode. The summation goes over all 15 normal vibrations of an octahedron. This parameter $\eta$ enters the equation of the equilibrium constant $K_{\mathrm{eq}}=n_{\mathrm{HS}} /\left(1-n_{\mathrm{HS}}\right)$ as follows:

$$
K_{\mathrm{eq}}(T)=\left(\frac{g_{\mathrm{HS}}}{g_{\mathrm{LS}}}\right)_{\mathrm{eq}} e^{-\frac{\Delta_{0}}{k_{\mathrm{B}} T}+\eta\left(n_{\mathrm{HS}}-\frac{1}{2}\right)}
$$

Here $\Delta$ denotes the energy difference between LS and HS states (in this approach independent on spin state of the lattice) and $g_{\mathrm{HS} / \mathrm{LS}}$ the degeneracies of a given spin state. A positive value of $\eta$ results in stabilization of the LS state below a critical temperature $\left(n_{\mathrm{HS}}=1 / 2\right)$ and its destabilization above it, i.e., corresponds to cooperative effect. We observe that $\left(\omega_{\mathrm{LS}}\right) \mathrm{HS}<\left(\omega_{\mathrm{LS}}\right) \mathrm{LS}$, while $\left(\omega_{\mathrm{HS}}\right) \mathrm{HS} \cong\left(\omega_{\mathrm{HS}}\right) \mathrm{LS}$, hence $\eta$ is negative for $\left(\left[\mathrm{Fe}(\mathrm{II})(4 \text {-amino-1,2,4-triazole })_{3}\right]^{2+}\right.$. This means that the vibrational coupling acts against a cooperative behavior, in line with the DFT predictions of [28], for which all normal vibrations were taken into account for the calculation of the vibrational entropy. In our opinion such a situation is more probable, at least at normal pressure.

\section{Conclusions}

We have presented a nuclear inelastic scattering and DFT study of the 1-D polymeric spin crossover 4-amino-1,2,4-triazole Fe(II) complexes which shows coupling of the vibrations involving the center of a given spin with the spin state of its neighbors. This coupling concerns both the energy of the vibration and its localization within the molecule. Particularly for the LS centers if the spin state of the neighbors is changed to HS the stretching of the remaining LS Fe(II) to lower frequencies and decoupling of the movement of its neighbors occur within the so modified mode. Similar effects are expected for the HS centers, whose vibrations may shift to higher frequencies upon change of spin of the neighbors to LS. The above mentioned frequency shifts are the likely reason of the predicted decrease of the vibrational contribution to the entropy upon a LLL $\rightarrow$ LHL to a HLH $\rightarrow$ HHH spin transition [28]. The observed spectra of the system at the temperature corresponding to $T_{\mathrm{c}}$ imply the clustering of the centers of the same spin rather than an alternate structure. Another, complementary method that could be used to investigate the distribution of the spin centers in the spin isomers, containing both LS and HS centers, as well as subtle structural phenomena, is the X-ray powder diffraction technique (cf. refs. [52,53]). Our DFT modeling also suggests, that for spin isomers containing comparable amounts of both spin isomers, independently on the distribution patterns, the HS and LS centers form two sublattices showing independent vibrations. Finally, the simulations of the experimentally obtained 1:1 mixture of HS and LS centers may imply that the structure of 1-D chains at $T_{\mathrm{C}}$ corresponds to a block (domain) pattern in one chain containing a 1:1 mixture of both spin isomers, rather than to corresponding alternate (chessboard) structures.

Supplementary Materials: The following are available online at www.mdpi.com/2312-7481/2/2/19/s1, GIF movies showing the discussed modes. The calculated sums of pDOS of all LS and all HS pentameric, heptameric, and nonameric models. 
Acknowledgments: I.F., J.A.W. and V.S. are grateful to the Allianz für Hochleistungsrechnen Rheinland-Pfalz (AHRP) for providing CPU-time within the projects TUKL-DFTRAMS and TUKL-NANOSCODFT. The financial support of German Ministry of Research and Education under contract number 05 K10UKA and of SPIN+X $\mathrm{SFB} / \mathrm{TRR} 173$ is gratefully acknowledged. We are grateful to Referees for their valuable comments and suggestions.

Author Contributions: V.S. and J.A.W. conceived and designed the experiments; J.A.W., I.F., J.M., A.I.C., R.R., K.S., H.-C.W. performed the experiments; I.F. and J.M performed data treatment, J.A.W. prepared the samples and performed DFT calculations; J.A.W. and V.S. wrote the paper.

Conflicts of Interest: The authors declare no conflict of interest.

\section{Abbreviations}

The following abbreviations are used in this manuscript:

$\begin{array}{ll}\text { DFT } & \text { Density Functional Theory } \\ \text { NIS } & \text { Nuclear Inelastic Scattering } \\ \text { SCO } & \text { Spin Crossover }\end{array}$

\section{References}

1. Garcia, Y.; Niel, V.; Muñoz, M.C.; Real, J.-A. Spin Crossover in 1D, 2D and 3D Polymeric Fe(II) Networks. Top. Curr. Chem. 2004, 233, 229-257.

2. Roubeau, O. Triazole-Based One-Dimensional Spin-Crossover Polymers. Chem. Eur. J. 2012, 18, 15230-15244. [CrossRef] [PubMed]

3. Gütlich, P.; Goodwin, H.A. Spin Crossover in Transition Metal Compounds I-III; Springer: Berlin Heidelberg, Germany, 2004.

4. Bousseksou, A.; Molnar, G.; Salmon, L.; Nicolazzi, W. Molecular spin crossover phenomenon: recent achievements and prospects. Chem. Soc. Rev. 2011, 40, 3313-3335. [CrossRef] [PubMed]

5. Gütlich, P. Spin Crossover-Quo Vadis? Eur. J. Inorg. Chem. 2013, 581-591. [CrossRef]

6. Halcrow, M., Ed.; Spin-Crossover Materials: Properties and Aplications; Wiley: Chichester, UK, 2013.

7. Kahn, O.; Kröber, J.; Jay, C. Spin Transition Molecular Materials for Displays and Data Recording. Adv. Mat. 1992, 4, 719-728. [CrossRef]

8. Kröber, J.; Audière, J.P.; Claude, R.; Codjovi, E.; Khan, O.; Haasnoot, J.G.; Grolière, F.; Jay, F.; Bousseksou, A.; Linares, J.; et al. Spin Transitions and Thermal Hysterseses in the Molecular-Based Materials $\left[\mathrm{Fe}(\mathrm{Htrz})_{2}(\operatorname{trz})\right]\left(\mathrm{BF}_{4}\right)$ and $\left[\mathrm{Fe}(\mathrm{Htrz})_{2}(\operatorname{trz})\right]\left(\mathrm{BF}_{4}\right) \cdot \mathrm{H}_{2} \mathrm{O}(\mathrm{Htrz}=1,2,4-4 \mathrm{H}$-triazole; trz = 1,2,4-triazolato). Chem. Mater. 1994, 6, 1404-1412. [CrossRef]

9. Kahn, O.; Jay-Martinez, C. Spin-Transition Polymers: From Molecular Materials Toward Memory Devices. Science 1998, 279, 44-48. [CrossRef]

10. Dîrtu, M.M.; Rotaru, A.; Gillard, D.; Linares, J.; Codjovi, E.; Tinant, B.; Garcia, Y. Prediction of the spin transition temperature in $\mathrm{Fe}(\mathrm{II})$ one-dimensional coordination polymers: An anion based database. Inorg. Chem. 2009, 48, 7838-7852. [CrossRef] [PubMed]

11. Lavrenova, L.G.; Shakirova, O.G.; Ikorskii, V.N.; Varnek, V.A.; Sheludyakova, L.A.; Larionov, S.V. ${ }^{1} A_{1} \rightarrow{ }^{5} T_{2}$ Spin Transition in New Thermochromic Iron(II) Complexes with 1,2,4-Triazole and 4-Amino-1,2,4-Triazole. Russ. J. Coord. Chem. 2003, 29, 24-30.

12. Dîrtu, M.M.; Schmidt, F.; Naik, D.A.; Rusu, I.; Rotaru, A.; Rackwitz, S.; Wolny, J.A.; Schünemann, V.; Spinu, L.; Garcia, B.Y. Two-Step Spin Transition in a 1D Fe ${ }^{\mathrm{II}}$ 1,2,4-Triazole Chain Compound. Chem. Eur. J. 2014, 21, 5843-5856. [CrossRef] [PubMed]

13. Müller, D.; Knoll, C.; Stöger, B.; Reissner, M.; Weinberger, P. A Modified Synthetic Pathway for the Synthesis of so far Inaccessible N1-Functionalized Tetrazole Ligands-Synthesis and Characterization of the 1D Chain-Type Spin Crossover Compound [Fe(3ditz) $)_{3}$ ] $\left(\mathrm{BF}_{4}\right)_{2}$. Eur. J. Inorg. Chem. 2013, 5-6, 984-991. [CrossRef] [PubMed]

14. Forestier, T.; Mornet, S.; Daro, N.; Nishihara, T.; Mouri, S.-I.; Tanaka, K.; Fouche, O.; Freysz, E.; Letard, J.-F. Nanoparticles of iron(II) spin-crossover. Chem. Commun. 2008, 4327-4329. [CrossRef] [PubMed]

15. Salmon, L.; Molnar, G.; Thibault, C.; Salmon, L.; Bousseksou, A.; Vieu, C. Soft lithographic patterning of spin crossover nanoparticles. Langmuir 2010, 26, 1557-1560. 
16. Tokarev, A.; Salmon, L.; Guari, Y.; Nicolazzi, W.; Molnar, G.; Bousseksou, A. Cooperative spin crossover phenomena in $\left[\mathrm{Fe}(\mathrm{NH} 2 \mathrm{trz})_{3}\right]$ (tosylate $)_{2}$ nanoparticles. Chem. Commun. 2010, 46, 8011-8013. [CrossRef] [PubMed]

17. Zitouni, D.; Quintero, C.; Bergaud, C.; Micheau, J.-C.; Bousseksou, A. A novel approach for fluorescent thermometry and thermal imaging purposes using spin crossover nanoparticles. J. Mater. Chem. 2010, 20, 5499-5503.

18. Coronado, E.; Galan-Mascaros, J.R.; Monrabal-Capilla, M.; Garcia-Martinez, J.; Pardo-Ibanez, P. Bistable Spin-Crossover Nanoparticles Showing Magnetic Thermal Hysteresis near Room Temperature. Adv. Mater. 2007, 19, 1359-1361. [CrossRef]

19. Galan-Mascaros, J.R.; Coronado, E.; Forment-Aliaga, A.; Monrabal-Capilla, M.; Pinilla-Cienfuegos, E.; Ceolin, M. Tuning size and thermal hysteresis in bistable spin crossover nanoparticles. Inorg. Chem. 2010, 49, 5706-5714. [CrossRef] [PubMed]

20. Tokarev, A.; Salmon, L.; Guari, Y.; Molnar, G.; Bousseksou, A. Synthesis of spin crossover nano-objects with different morphologies and properties. New. J. Chem. 2011, 35, 2081-2088. [CrossRef]

21. Forestier, T.; Mornet, S.; Kaiba, A.; Pechev, S.; Denux, D.; Guionneau, P.; Daro, N.; Nishihara, T.; Mouri, S.-I.; Tanaka, K.; et al. Nanoparticles of $\left[\mathrm{Fe}\left(\mathrm{NH}_{2}-\mathrm{trz}_{3}\right] \mathrm{Br}_{2} 3 \mathrm{H}_{2} \mathrm{O}\left(\mathrm{NH}_{2}\right.\right.$-trz $=4$-amino-1,2,4-triazole $)$ prepared by the reverse micelle technique: Influence of particle and coherent domain size on spin-crossover properties. Chem. Eur. J. 2009, 25, 6122-6130. [CrossRef] [PubMed]

22. Enanescu, C.; Nishino, M.; Miyashita, S. Spin-Crossover Materials; Halcrow, M., Ed.; Wiley: Chichester, UK, 2013.

23. Gudyma, I.; Maksymov, A.; Enanescu, C. Phase transition in spin-crossover compounds in the breathing crystal field model. Phys. Rev. B 2014, 89, 224412-224419. [CrossRef]

24. Spiering, H. Elastic Interaction in Spin Crossover Compounds. Top. Curr. Chem. 2004, 235, 171-195.

25. Grosjean, A.; Daro, N.; Pechev, S.; Moulet, L.; Etrillard, C.; Chastanet, G.; Guinneau, P. The Spin-Crossover Phenomenon at the Coherent-Domains Scale in 1D Polymeric Powders: Evidence for a Structural Fatigability. Eur. J. Inorg. Chem. 2016. [CrossRef]

26. Linares, J.; Spiering, H.; Varret, F. Analytical solution of 1D Ising-like systems modified by weak long range interaction. Eur. J. Phys. B 1999, 10, 271-275.

27. Klokishner, S.; Linares, J.; Varret, F. Effect of hydrostatic pressure on phase transitions in spin-crossover 1D systems. Chem. Phys. 2000, 255, 317-323. [CrossRef]

28. Rackwitz, S.; Klopper, W.; Schünemann, V.; Wolny, J.A. Quantification of intramolecular cooperativity in polynuclear spin crossover Fe(II) complexes by density functional theory calculations. Phys. Chem. Chem. Phys. 2013, 15, 15450-15458. [CrossRef] [PubMed]

29. Rackwitz, S.; Wolny, J.A.; Muffler, K.; Achterhold, K.; Rüffer, R.; Garcia, Y.; Diller, R.; Schünemann, V. Vibrational properties of the polymeric spin crossover (SCO) Fe(II) complexes [\{Fe(4-amino-1,2,4-triazole $\left.\left.)_{3}\right\} \mathrm{X}_{2}\right]_{\mathrm{n}}$ : A nuclear inelastic scattering (NIS), Raman and DFT study. Phys. Chem. Chem. Phys. 2012, 14, 14650-14660. [CrossRef] [PubMed]

30. Wolny, J.A.; Diller, R.; Schünemann, V. Vibrational Spectroscopy of Mono- and Polynuclear Spin-Crossover Systems. Eur. J. Inorg. Chem. 2012, 16, 2635-2648. [CrossRef]

31. Felix, G.; Mikolasek, M.; Peng, H.; Nicolazzi, W.; Molnar, G.; Chumakov, A.I.; Salmon, L.; Bousskesou, A. Lattice dynamics in spin-crossover nanoparticles through nuclear inelastic scattering. Phys. Rev. B. 2015, 91, 024422-024425.

32. Wolny, J.A.; Rackwitz, S.; Chumakov, A.I.; Faus, I.; Huang, H.; Rüffer, R.; Schlage, K.; Wille, H.-C.; Schünemann, V. Experimental evidence of the vibrational coupling of nearest neighbours in 1D spin crossover polymers of rigid bridging ligands. Hyperfine Inter. 2014, 226, 193-197. [CrossRef]

33. Wolny, J.A.; Rackwitz, S.; Achterhold, K.; Muffler, K.; Schünemann, V. Nuclear inelastic scattering of 1D polymeric $\mathrm{Fe}(\mathrm{II})$ complexes of 1,2,4-aminotriazole in their high-spin and low-spin state. Hyperfine Interact. 2012, 204, 129-132. [CrossRef]

34. Reiher, M.; Salomon, O.; Hess, B.A. Reparametrization of hybrid functionals based on energy differences of states of different multiplicity. Theor. Chim. Act. 2001, 107, 48-55. [CrossRef]

35. Salomon, O.; Reiher, M.; Hess, B.A. Assertion and validation of the performance of the B3LYP functional for the first transition metal row and the G2 test set. J. Chem. Phys. 2002, 117, 4729-4737. [CrossRef] 
36. Stevens, W.J.; Basch, H.; Krauss, J. Compact effective potentials and efficient shared-exponent basis-sets for the 1st-row and 2nd-row atoms. J. Phys. Chem. 1984, 81, 6026-6033. [CrossRef]

37. Stevens, W.J.; Krauss, M.; Basch, H.; Jasien, P.G. Relativistic compact effective potentials and efficient, shared-exponent basis-sets for the 3rd-row, 4th-row, and 5th-row atoms. Can. J. Chem. 1992, 70, 612-630.

38. Cundari, T.R.; Stevens, W.J. Effective core potential methods for the lanthanides. J. Chem. Phys. 1993, 98, 5555-5565. [CrossRef]

39. Frisch, M.J.; Trucks, G.W.; Schlegel, H.B.; Scuseria, G.E.; Robb, M.A.; Cheeseman, J.R.; Scalmani, G.; Barone, V.; Mennucci, B.; Petersson, G.A. Gaussian 09, Revision D.01; Gaussian: Wallingford, CT, USA, 2013.

40. Paulsen, H.; Winkler, H.; Trautwein, A.X.; Grünstreudel, H.; Rusanov, V.; Toftlund, H. Measurement and simulation of nuclear inelastic-scattering spectra of molecular crystals. Phys. Rev B. 1999, 59, 975-984. [CrossRef]

41. Murakami, Y.; Komatsu, T.; Kojima, N. Control of Tc and spin bistability in the spin-crossover system, $\left[\mathrm{Fe}\left(4-\mathrm{NH}_{2} \mathrm{trz}\right)_{3}\right]\left(\mathrm{R}-\mathrm{SO}_{3}\right)_{2}$. Synth. Metals 1999, 103, 2157-2158. [CrossRef]

42. Codjovi, E.; Sommier, L.; Kahn, O.; Jay, C. A spin transition molecular material with an exceptionally large thermal hystersisi loop at room temperature. New J. Chem. 1996, 20, 503-505.

43. Sorai, M. Heat Capacity Studies of Spin Crossover Systems. Top. Curr. Chem. 2004, 235, 153-170.

44. Sorai, M.; Ensling, J.; Gütlich, P. Mössbauer Effect Study on Low Spin ${ }^{1} \mathrm{~A}_{1}<->$ High-Spin ${ }^{5} \mathrm{~T}_{2}$ Transition in tris(2-picolylamine) iron chloride I. Dilution Effect in $\left[\mathrm{Fe}_{x} \mathrm{Zn}_{1-x}(2-\text { pic) })_{3}\right] \mathrm{Cl}_{2} \mathrm{C}_{2} \mathrm{H}_{5} \mathrm{OH}$. Chem. Phys. 1976, 19, 199-209.

45. Molnár, T.; Galetm, A.; Molnár, G.; Carmen Munoz, M.; Zwick, A.; Tanaka, K.; Real, J.-A.; Bousseksou, A. Metal Dilution Effects on the Spin-Crossover Properties of the Three-Dimensional Coordination Polymer Fe(pyrazine) $\left[\mathrm{Pt}(\mathrm{CN})_{4}\right]$. J. Phys.Chem. B 2005, 109, 14859-14867.

46. Tuchagues, J.-P.; Bousseksou, A.; Molnár, G.; McGarvey, J.J.; Varret, F. The Role of Molecular Vibrations in the Spin Crossover Phenomenon. Top. Curr. Chem. 2004, 235, 85-103.

47. Benda, R.; Bousseksou, A.; Chumakov, A.I.; Dennis, A.; Hert, C.; Gütlich, P.; Höfer, A.; McGarvey, J.J.; Paulsen, H.; Ronayne, K.L.; et al. Vibrational spectrum of the spin crossover complex [Fe(phen $\left.)_{2}(\mathrm{NCS})_{2}\right]$ studied by IR and Raman spectroscopy, nuclear inelastic scattering and DFT calculations. Phys. Chem. Chem. Phys. 2006, 8, 4685-4693.

48. Paulsen, H.; Schünemann, V.; Wolny, J. Progress in Electronic Structure Calculations on Spin-Crossover Complexes. Eur. J. Inorg. Chem. 2013, 628-641. [CrossRef]

49. Zimmerman, R.; König, E. A Model for High-Spin/Low-Spin Transitions in Solids Including the Effect of Lattice Vibrations. J. Phys. Chem. Solids 1977, 38, 779-798. [CrossRef]

50. Spiering, H.; Boukhaddaden, K.; Linares, J.; Varret, F. Total free energy of a spin-crossover molecular system. Phys. Rev. B 2004, 70, 184106-184115. [CrossRef]

51. Bousskesou, A.; Constant-Machado, H.; Varret, F. A Simple Ising-Like Model for Spin Conversion Including Molecular Vibration. J. Phys. I France 1995, 5, 747-760. [CrossRef]

52. Chernyshov, D.; Hostettler, M.; Törnroos, K.W.; Bürgi, H.-B. Ordering Phenomena and Phase Transitions in a Spin-Crossover Compound-Uncovering the Nature of the Intermediate Phase of $\left[\mathrm{Fe}(2-\mathrm{pic})_{3}\right] \mathrm{Cl}_{2}-\mathrm{EtOH}$. Angew. Chem. Int. Ed. 2003, 42, 3825-3830. [CrossRef] [PubMed]

53. Neville, S.M.; Halder, G.J.; Chapman, W.; Duriska, M.B.; Moubaraki, B.; Murray, K.S.; Kepert, C.J. Guest tunable structure and spin crossover properties in a nanoporous coordination framework material. J. Am. Chem. Soc. 2009, 131, 12106-12108. [CrossRef] [PubMed]

(C) 2016 by the authors; licensee MDPI, Basel, Switzerland. This article is an open access article distributed under the terms and conditions of the Creative Commons by Attribution (CC-BY) license (http://creativecommons.org/licenses/by/4.0/). 